\title{
Chemotherapy for Childhood Acute Myeloid Leukemia and Associated Infections Over Two Decades in India: Timeline and Impact on Outcome
}

\begin{abstract}
Background: Infection and relapse constitute the two main challenges in the management of acute myeloid leukemia (AML) in children. Real-world data in children treated in low-and-middle income countries are sparse as the cost of supportive care is high. Patients and Methods: We present data on children up to 18 years of age undergoing chemotherapy for AML as per UKMRC AML protocol from 2002 to June 2019 and pattern of sepsis. Results: The incidence of culture-positive sepsis was similar pre- and post-2012 (52.6\% vs. $72.4 \%)$, Klebsiella pneumoniae being the most common organism. There was a significant increase in carbapenem resistance post $2012(14 \%$ vs. $67 \%, P=0.032$ ). Sepsis-related induction mortality has remained at $6.2 \%$ despite an increase in drug-resistant bacterial infections over two decades. The overall survival was $53 \%(\mathrm{n}=48)$, with a plateau in the survival curve after 24 months, relapse being the most common cause of death $(69 \%)$. Conclusions: Sepsis-related induction mortality can be maintained at less than $10 \%$ in children undergoing chemotherapy for AML, despite increasing drug-resistant bacteremia, with adequate supportive care and trained personnel including pediatric intensivists and nurses.
\end{abstract}

Keywords: Childhood acute myeloid leukemia, drug-resistant sepsis, hematopoietic stem cell transplantation, relapse, sepsis-related mortality

\section{Introduction}

High-dose chemotherapy is the backbone of acute myeloid leukemia (AML) requiring significant supportive care. ${ }^{[1,2]}$ Sepsis has been a considerable obstacle in providing optimal care for AML chemotherapy, and the associated cost adds to the already strained finances of families. ${ }^{[3]}$ Over the last few decades, hospital infection control policies (HIC) have had to grapple with changing hospital flora and the spectrum of evolving bacterial species, particularly multidrug-resistant organisms (MDROs). We had documented a sudden increase in the incidence and isolation of drug-resistant gram-negative bacterial sepsis after 2012. We aimed to analyze the changing spectrum in bacteremia over 18 years and the impact that emerging drug-resistant organisms have had on the outcome.

\section{Patients and Methods}

A retrospective observational study was performed in the department of pediatric hematooncology, blood and marrow transplantation. We included all children

This is an open access journal, and articles are distributed under the terms of the Creative Commons Attribution-NonCommercial-ShareAlike 4.0 License, which allows others to remix, tweak, and build upon the work non-commercially, as long as appropriate credit is given and the new creations are licensed under the identical terms.

For reprints contact: WKHLRPMedknow_reprints@wolterskluwer.com up to 18 years of age diagnosed to have AML at our center and who received chemotherapy from January 2002 to June 2019. Exclusion criteria included children who proceeded to upfront hematopoietic stem cell transplantation (HSCT), those with infant AML, those diagnosed to have AML M3 with $t(15 ; 17)$ or variants, and those diagnosed to have mixed phenotype acute leukemia. The diagnosis of AML was confirmed through flow cytometry to determine leukemia-associated immunophenotype. We performed fluorescence in situ hybridization and karyotyping in all diagnostic bone marrow samples. The treatment was risk-stratified, based on molecular markers.

All children received treatment as per the UKMRC AML 15 protocol, including two cycles of induction (cytarabine, daunorubicin, and etoposide) followed by two cycles of consolidation (high-dose cytarabine). We performed a bone marrow assessment at the end of the first induction for remission status and following the second consolidation for the end of treatment assessment. We performed a repeat bone

How to cite this article: Uppuluri R, Swaminathan VV,
Ravichandran N, Ramanan KM, Meena S, Varla H,
et al. Chemotherapy for childhood acute myeloid
leukemia and associated infections over two decades
in India: Timeline and impact on outcome. Indian J
Med Paediatr Oncol 2020;41:869-73.

\author{
Ramya Uppuluri' ${ }^{1}$, \\ Venkateswaran \\ Vellaichamy \\ Swaminathan', \\ Nikila
}

Ravichandran ${ }^{1}$, Kesavan Melarcode Ramanan', Satishkumar Meena1, Harika Varla ${ }^{1}$, Balasubramaniam Ramakrishnan ${ }^{1}$, Indira Jayakumar², Revathi Raj ${ }^{1}$

${ }^{1}$ Department of Pediatric Hematology, Oncology, Blood and Marrow Transplantation, Apollo Hospitals, Chennai, Tamil Nadu, India, ${ }^{2}$ Department of Pediatric Critical Care, Apollo Hospitals, Chennai, Tamil Nadu, India

Submitted: 02-May-2020 Revised: 16-Jul-2020 Accepted: 02-Oct-2020 Published: 31-Dec-2020

Address for correspondence: Dr. Ramya Uppuluri, Department of Pediatric Hematology, Oncology, Blood and Marrow Transplantation, Apollo Hospitals, 320 , Padma Complex, Anna Salai, Teynampet, Chennai - 600035 , Tamil Nadu, India.

E-mail: ramya.december@ gmail.com

Access this article online

Website: www.ijmpo.org

DOI: 10.4103/ijmpo.ijmpo_211_20 Quick Response Code:

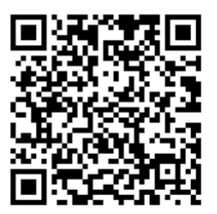


marrow assessment at the end of the second induction for those with minimal residual disease (MRD) positive disease after induction cycle 1 . An evaluation was done through examination for morphological remission alone up until 2009. From 2009, we performed MRD assessment by flow cytometry for all children at all time points. As per the protocol, MRD of $<0.1 \%$ was negative. We offered HSCT if the child had high-risk cytogenetics, persistently positive MRD, refractory disease, and relapsed disease.

We continued antifungal, antiviral, and pneumocystis carinii prophylaxis for all children throughout chemotherapy. Antifungal prophylaxis included fluconazole up until 2012. Since 2012, we have consistently used micafungin as antifungal prophylaxis. In the children with proven fungal infection in the previous cycle, we used voriconazole or amphotericin as prophylaxis. All children received granulocyte-colony stimulating factor from day 7 after completion of chemotherapy.

Febrile neutropenia was treated with antibiotics as per the HIC policy after taking adequate samples for cultures. We followed a policy of de-escalation of antibiotics when the child was afebrile for $48 \mathrm{~h}$, and the blood cultures were sterile. Antibiotic policy was based on the HIC and the existing antibiogram. A combination of third generation cephalosporin with beta-lactamase inhibitor was the most frequent first-line antibacterial agent used. A carbapenem with or without teicoplanin for Gram-positive cover was used in case of persistent febrile neutropenia. The decision was also based on the presence or absence of any specific focus for infection and the anticipated duration of neutropenia. All culture-positive bacteremia was treated with antibiotics based on the drug sensitivity after consulting the infectious disease specialists.

We performed stool sample screening for the presence of carbapenem resistance through a multiplex polymerase chain reaction. We introduced colistin early in the children with resistant pathogens on stool screening. In children with suspected central line-associated bacteremia, we performed an additional peripheral blood culture. In case of persistent fever for over $72 \mathrm{~h}$ or if there were localizing signs, we performed early imaging.

We analyzed the data on the demography, subclassification of AML, remission status, rate of relapse, treatment postrelapse, infectious complications, species isolated and drug sensitivity, rates of fungal infections, and the associated morbidity and mortality. The data on infections were analyzed pre-and post-2012. A septic event was the detection of culture-proven bacteremia. Written informed consent was obtained from the parents of all children, and the institutional ethics review board approved the study.

\section{Statistical methods}

Continuous variables were represented as mean \pm standard deviation or median, if they were normally or nonnormally distributed. Categorical variables were expressed as percentage. Comparison of normally distributed continuous variables was done by independent sample $t$-test and of nonnormally distributed continuous variables by MannWhitney test. Categorical variables were compared by either Chi-square test or Fisher's exact test based on the number of observations. Kaplan-Meier curve was drawn to assess the survival pattern with log rank test for comparison of survival pattern. Data entry was done into MS excel sheet, with validation and analysis by SPSS version 25.0 (Armonk, NY: IBM Corp.). All $P<0.05$ were considered as statistically significant.

\section{Results}

We included a total of 48 children with an equal male: female ratio; median age of 8 years. The predominant subtype of AML was M2 in 34 children (70\%), followed by M4 in $9(18 \%), M 1$ in $2(4 \%), M 6$, M3 with $t(17 ; 17)$ and myeloid sarcoma stage 4 in one child each.

\section{Culture-positive bacteremia}

Thirty-one (64\%) children developed culture-positive bacteremia and sepsis during chemotherapy. The septic events were predominantly noted during induction chemotherapy in 28/31 (90\%), and during consolidation in $3 / 31(10 \%)$ children. Of the $31,25(80 \%)$ were gram-negative bacilli (GNB) while 6 (20\%) were Gram-positive cocci (GPC). Among the GNB, the most common organism was Klebsiella pneumoniae in 18/25 (72\%), Escherichia coli in 5/25 (20\%), Pseudomonas aeruginosa in $1 / 25$, and Vibrio cholerae in $1 / 25$. We documented GPC in six children-3/6 Streptococcus viridans, 2/6 coagulase-negative Staphylococcus aureus, and 1/6 methicillin-sensitive $S$. aureus. Among the GNB, 4/5 E. coli (80\%) and 10/18 $(55 \%)$ of the $K$. pneumoniae were carbapenem-resistant. All children with carbapenem-resistant bacteremia received colistin. The focus for infection was predominantly gut in $26 / 31(83 \%)$. We had documented that perianal ulcers preceded GNB bacteremia in $20 / 31$ cases $(64 \%)$, and this was statistically significant $(P$ value 0.001$)$.

\section{Pre-and post-2012}

Nineteen children underwent treatment before 2012, and 29 children underwent treatment post-2012. Ten $(52.6 \%)$ developed culture-proven bacteremia prior to 2012, while 21 (72.4\%) developed post 2012. K. pneumoniae has remained the predominant organism throughout. We documented carbapenem-resistance in only one child before 2012. However, after 2012, we had documented 13 children with carbapenem-resistance. There was a documented increase in carbapenem-resistance post 2012, which was statistically significant $(14 \%$ vs. $67 \%, P=0.032)$.

\section{Fungal infections}

We documented fungal infections in $7 / 48$ (14.5\%). Proven fungal infection with the isolation of candida species in 
the bloodstream was recorded in one child (2\%), while 6 $(12.5 \%)$ had probable fungal infections, localized in the lung.

\section{Interventions to combat severe sepsis}

Febrile neutropenia necessitated admission to the pediatric critical care unit (PICU) in $32(66 \%)$ children. Of the 32 admissions, 28 (87\%) children were discharged from PICU when they recovered from septic events. Early granulocyte transfusion administered in 13/32 (40\%) children prevented organ damage. Granulocyte transfusions were indicated in those with neutropenia and culture positive sepsis with or without features of early septic shock and where neutropenia was not expected to recover within the next $48 \mathrm{~h}$. Central lines were removed and changed soon in all children demonstrating features of sepsis before the onset of septic shock. Monitoring and supportive care for mucositis, Sitz bath for perianal care twice a day reduced bacterial translocation. Meticulous perianal hygiene involved the use of a barrier cream of lanolin ointment alternating with "triple cream," a combination of clotrimazole, mupirocin, and zinc oxide. We had a unit policy for neutropenic diet, enteral nutrition, including the use of protein supplements and antibiotic stewardship as per the HIC team.

\section{Outcome and survival}

Overall survival in our cohort was $53 \%$ with a median follow up of 85 months. The mean survival time was 95

\begin{tabular}{lc}
\hline \multicolumn{1}{c}{ Table 1: Causes of death in the cohort } \\
\hline Cause of death $(\boldsymbol{n = 2 3 / 4 8 ; ~ 4 7 \% )}$ & $\begin{array}{c}\boldsymbol{n} \text { (percentage } \\
\text { of total 48) }\end{array}$ \\
\hline Disease relapse & $15(31)$ \\
Disease progression & $3(6.2)$ \\
Induction sepsis & $3(6.2)$ \\
Sepsis post-HSCT & $1(2)$ \\
Uncontrolled seizures 1-year posttreatment & $1(2)$ \\
\hline
\end{tabular}

HSCT: Hematopoietic stem cell transplantation

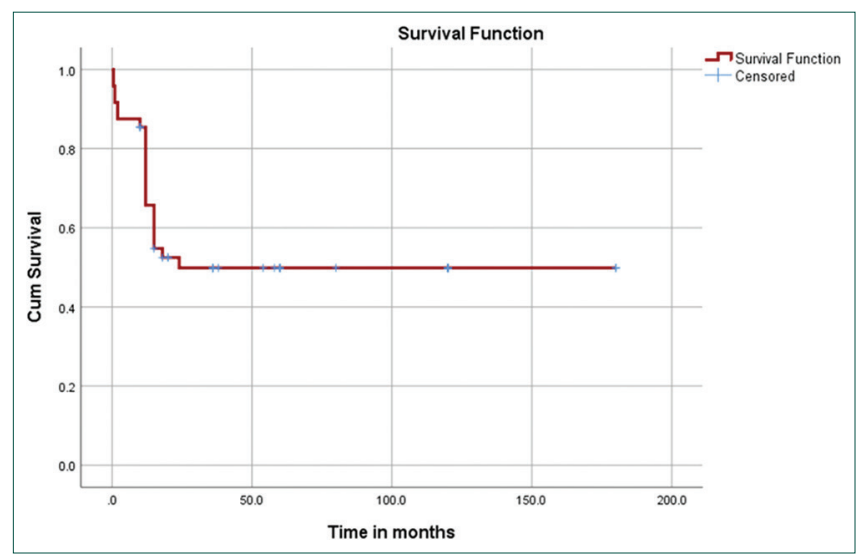

Figure 1: Kaplan-Meier survival curve analysis demonstrating overall survival of $53 \%$ with mean survival time was 95 months (confidence interval 95\% 70.4-119 months), with a striking plateau in the survival curve after 24 months months (confidence interval [CI] 95\% 70.4 months-119 months), with a plateau in the survival curve after 24 months [Figure 1]. Causes of death are shown in Table 1. Overall, the induction mortality due to sepsis was $6.2 \%$. Of the three children who died of sepsis during induction, two were treated prior to 2012 and one child post 2012. Among the 17 children who did not develop culture proven bacteremia, $10(58 \%)$ are alive and in remission, while 7 died due to disease relapse.

The relapse rate in our cohort was 20/48 (41\%). HSCT was performed in five children who relapsed, one matched sibling donor HSCT and two each undergoing matched unrelated donor and unmanipulated $\mathrm{T}$ replete haploidentical transplantation with posttransplant cyclophosphamide, respectively. Four children are alive and in remission $(80 \%)$, while one child died of sepsis before engraftment. HSCT had a statistically significant survival advantage with mean survival time post-HSCT of 66 months (CI 95\% 42-90) as against 14 months (CI 95\% 12.3-15.8) among those not transplanted ( $P$ value 0.002) [Figure 2]. All children who relapsed and did not undergo transplant succumbed to progressive disease.

\section{Discussion}

Our study reports a significant increase in carbapenem resistance among GNB post-2012. However, the induction mortality due to sepsis has remained low and constant over two decades, with overall survival of $53 \%$. The myelodysplasia-related changes AML-10 trials published their results with overall survival of $57 \%$. Infections, mainly fungal, were a predominant cause of death. ${ }^{[4]}$

Several groups over the years have reported GPCs to be the predominant organisms isolated, up until 2016. In a publication from 2007, where 492 children enrolled in the CCG2961 study were analyzed for infection-related mortality (IRM), fungi and GPCs were most commonly associated. ${ }^{[3]}$ Similar results were published from Italy in

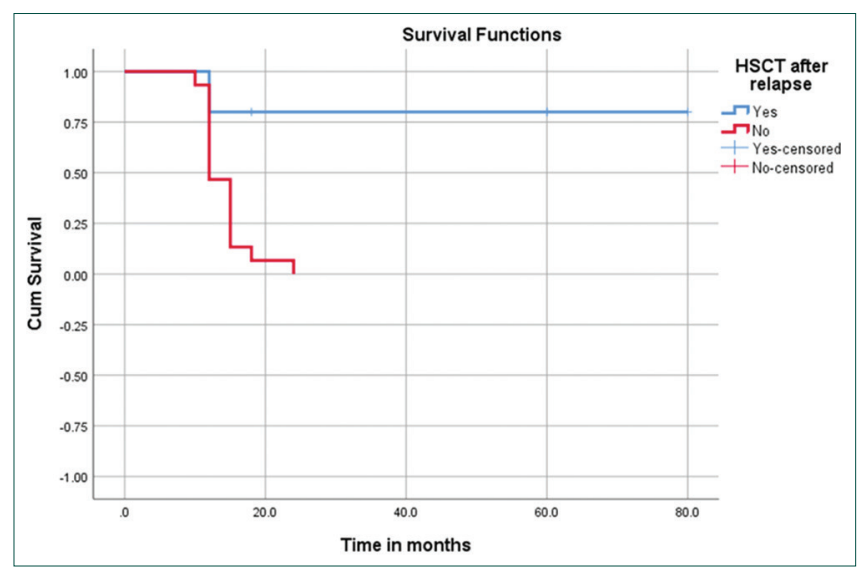

Figure 2: Kaplan-Meier survival curve analysis with mean survival time post- hematopoietic stem cell transplantation of 66 months (confidence interval $95 \% 42-90$ ) as against 14 months (confidence interval $95 \%$ 12.3-15.8) among those not transplanted $(P=0.002)$ 
2010, where 240 children undergoing AML chemotherapy were analyzed with bacteremia noted in $32 \%$, GPCs being the predominant organisms isolated..$^{[5]}$ Bochennek et al. published data on infections during chemotherapy with AML-BFM 2004 protocol, where isolates were predominantly GPCs as against GNBs (240 vs. 90 ), with an IRM of $5.4 \%{ }^{[6]}$

There has been, however, a global change in the patterns of MDROs being isolated. In 2019, a group from Germany reported inferior outcomes in patients colonized with carbapenem-resistant organisms among those undergoing AML chemotherapy. ${ }^{[7]}$ Similar results have also been reported from India in 2018, where Jaiswal et al. demonstrated poor outcomes in adults undergoing chemotherapy who were colonized with carbapenem-resistant enterobacteriaceae. ${ }^{[8]}$ In 2016, a multicenter study from Italy found that of the K. pneumoniae isolates among patients with hematological malignancies, $57.9 \%$ were carbapenem-resistant and contributed significantly to mortality. ${ }^{[9]}$ In another study from Italy, among the patients with carbapenem-resistant Klebsiella, mortality was highest in those with AML. ${ }^{[10]}$ In 2019, a multicenter study from India analyzed over 5000 isolates and found that $E$. coli and $K$. pneumoniae were isolated in $37 \%$ and $26 \%$, respectively. Mortality was higher among those with MDROs. ${ }^{[1]}$

Fungal infections were not the predominant cause of morbidity or mortality in our cohort, as noted in $14 \%$ of children with only one child diagnosed to have a proven fungal infection. This is in contrast to several groups worldwide reporting invasive fungal infections as an essential contributor to infection rates. ${ }^{[3,4]}$ In 2019, Patel et al. reported invasive mold infections in 28/162 adults undergoing AML chemotherapy. ${ }^{[12]}$ The use of micafungin in prophylaxis and early imaging and intervention has helped keep the incidence of fungal sepsis low in our cohort.

Hand hygiene, frequent surface cleaning, isolation, and antibiotic stewardship are essential to contain MDROs. ${ }^{[13,14]}$ In our cohort, early intervention with the pediatric intensive care team in sepsis-related events, including granulocyte transfusions, has a positive impact on survival. ${ }^{[15]}$

Limitations of the study include the retrospective nature, the lack of a control group and the nonavailability of MRD assessment prior to 2009 .

\section{Conclusions}

Despite changes in the bacterial isolates over two decades and increase in carbapenem-resistant organisms, adequate infection control and treatment by a team including pediatric oncologists, pediatric intensivists, infectious disease specialists, and oncology nurses helps keep sepsis related mortality down to $<10 \%$ in children undergoing chemotherapy for AML, with an overall survival of $53 \%$.
The bigger challenge is the relapse rate which has remained at around $30 \%$, particularly in the first 2 years' post treatment. HSCT provides a significant survival advantage in relapsed AML. Precise risk stratification and safer HSCT techniques are the need of the hour for children with AML.

\section{Acknowledgments}

We would like to acknowledge the immense support provided by the departments of hematology, stem cell apheresis, and infectious disease specialists in the care of these children.

\section{Financial support and sponsorship}

Nil.

\section{Conflicts of interest}

There are no conflicts of interest.

\section{References}

1. Löwenberg B, Downing JR, Burnett A. Acute myeloid leukemia. N Engl J Med 1999;341:1051-62.

2. Atallah E, Cortes J, O'Brien S, Pierce S, Rios MB, Estey E, et al. Establishment of baseline toxicity expectations with standard frontline chemotherapy in acute myelogenous leukemia. Blood 2007;110:3547-51.

3. Sung L, Lange BJ, Gerbing RB, Alonzo TA, Feusner J. Microbiologically documented infections and infection-related mortality in children with acute myeloid leukemia. Blood 2007;110:3532-9.

4. Riley LC, Hann IM, Wheatley K, Stevens RF. Treatment-related deaths during induction and first remission of acute myeloid leukaemia in children treated on the Tenth Medical Research Council acute myeloid leukaemia trial (MRC AML10). The MCR Childhood Leukaemia Working Party. Br J Haematol 1999; 106:436-44.

5. Castagnola E, Rossi MR, Cesaro S, Livadiotti S, Giacchino M, Zanazzo $\mathrm{G}$, et al. Incidence of bacteremias and invasive mycoses in children with acute non-lymphoblastic leukemia: Results from a multi-center Italian study. Pediatr Blood Cancer 2010;55:1103-7.

6. Bochennek K, Hassler A, Perner C, Gilfert J, Schöning S, Klingebiel $\mathrm{T}$, et al. Infectious complications in children with acute myeloid leukemia: Decreased mortality in multicenter trial AML-BFM 2004. Blood Cancer J 2016;6:e382.

7. Ballo O, Tarazzit I, Stratmann J, Reinheimer C, Hogardt M, Wichelhaus TA, et al. Colonization with multidrug resistant organisms determines the clinical course of patients with acute myeloid leukemia undergoing intensive induction chemotherapy. PLoS One 2019;14:e210991.

8. Jaiswal SR, Gupta S, Kumar RS, Sherawat A, Rajoreya A, Dash SK, et al. Gut Colonization with Carbapenem-resistant Enterobacteriaceae Adversely Impacts the Outcome in Patients with Hematological Malignancies: Results of A Prospective Surveillance Study. Mediterr J Hematol Infect Dis 2018;10:e2018025.

9. Trecarichi EM, Pagano L, Martino B, Candoni A, Di Blasi R, Nadali G, et al. Bloodstream infections caused by klebsiella pneumonia in onco-hematological patients: Clinical impact of carbapenem resistance in a multicentre prospective survey. Am J Hematol 2016;91:1076-81.

10. Micozzi A, Gentile G, Minotti C, Cartoni C, Capria S, Ballaro D, 
et al. Carbapenem-resistant klebsiella pneumonia in high-risk hematological patients: Factors favoring spread, risk factors and outcome of carbapenem-resistant klebsiella pneumonia bacteremias. BMC Infect Dis 2017;17:203.

11. Gandra S, Tseng KK, Arora A, Bhowmik B, Robinson ML, Panigrahi $\mathrm{B}$, et al. The mortality burden of multidrug-resistant pathogens in India: A retrospective, observational study. Clin Infect Dis 2019;69:563-70.

12. Patel HP, Perissinotti AJ, Patel TS, Bixby DL, Marshall VD, Marini BL. Incidence and risk factors for breakthrough invasive mold infections in acute myeloid leukemia patients receiving remission induction chemotherapy. Open Forum Infect Dis 2019;6:ofz176.

13. Haque M, Sartelli M, McKimm J, Abu Bakar M. Health care-associated infections An overview. Infect Drug Resist 2018;11:2321-33.

14. Trubiano JA, Worth LJ, Thursky KA, Slavin MA. The prevention and management of infections due to multidrug-resistant organisms in hematology patients. $\mathrm{Br} \mathrm{J}$ Clin Pharmacol 2015;79:195-207.

15. Uppuluri R, Ramachandrakurup S, Vaidhyanathan L, Kandath S, Subburaj D, Raj R. Changing trends in the use of granulocyte transfusions in neutropenic children with sepsis in India. Indian J Hematol Blood Transfus 2017;33:207-10. 